\title{
Planejamento estratégico de ações educativas sobre Hipertensão e Diabetes na
}

\section{Atenção Básica: Relato de experiência}

\author{
Strategic planning of educational actions on Hypertension and Diabetes in Primary Care: \\ Experience report \\ Planificación estratégica de acciones educativas sobre Hipertensión y Diabetes en Atención \\ Primaria: Informe de experiência
}

Recebido: 13/07/2021 | Revisado: 19/07/2021 | Aceito: 25/07/2021 | Publicado: 01/08/2021

\author{
Walleska Ferreira Turques \\ ORCID: https://orcid.org/0000-0002-9655-8011 \\ Universidade Federal Fluminense, Brasil \\ E-mail: walleska_turques@id.uff.br \\ Jéssica Santos de Souza Leal \\ ORCID: https://orcid.org/0000-0002-9215-6090 \\ Universidade Federal Fluminense, Brasil \\ E-mail: jleal0803@gmail.com \\ Thicianne de Lucena Siqueira Vieira \\ ORCID: https://orcid.org/0000-0002-4484-5860 \\ Universidade Federal Fluminense, Brasil \\ E-mail: thiciannelucena@id.uff.br \\ Maritza Consuelo Ortiz Sanchez \\ ORCID: https://orcid.org/0000-0002-6123-9846 \\ Universidade Federal Fluminense, Brasil \\ E-mail: morsa_peru@yahoo.com \\ Érica Brandão de Moraes \\ ORCID: https://orcid.org/0000-0003-3052-158X \\ Universidade Federal Fluminense, Brasil \\ E-mail: ericabrandao@id.uff.br \\ André Luís de Souza Braga \\ ORCID: https://orcid.org/0000-0002-7961-9038 \\ Universidade Federal Fluminense, Brasil \\ E-mail: andre.braga @globo.com \\ Miriam Marinho Chrizostimo \\ ORCID: https://orcid.org/0000-0001-7498-4637 \\ Universidade Federal Fluminense, Brasil \\ E-mail: miriammarinho@hotmail.com \\ Maria Lelita Xavier \\ ORCID: https://orcid.org/0000-003-3014-733X \\ Universidade do Estado do Rio de Janeiro, Brasil \\ E-mail: lely108@hotmail.com
}

\begin{abstract}
Resumo
Objetivo: Relatar a experiência vivenciada no planejamento das ações educativas sobre Hipertensão e diabetes realizada com idosos na atenção básica. Método: O presente estudo de natureza descritiva trata de um relato de experiência sobre a utilização da roda de conversa como estratégia de planejamento de atividade de educação em saúde com usuários idosos, no contexto do Ensino Teórico Prático da disciplina de Gerência de Enfermagem I, no primeiro semestre de 2019 em uma unidade de Saúde no município de Niterói-RJ, vinculada ao Sistema Único de Saúde. Resultados: A roda de conversa é uma estratégia satisfatória visto que o público-alvo que vive com a hipertensão e diabetes teve a oportunidade de expandir suas perspectivas para tomada de melhores decisões e melhorar sua qualidade de vida decorrente da troca de conhecimento de temas de interesse dos usuários. Conclusão: O planejamento das atividades da roda de conversa como estratégia para atingir os objetivos de promover a saúde dos idosos hipertensos e diabéticos, propiciou participação ativa dos mesmos, com trocas de informações que visam o desenvolvimento da autonomia do indivíduo e a redução dos danos. No que diz respeito aos estudantes trouxe clareza de seu papel no planejamento e organização de sua prática, além de maior segurança em suas abordagens enquanto futuros profissionais responsáveis pela educação em saúde do usuário e comunidade.
\end{abstract}

Palavras chave: Planejamento em saúde; Educação em saúde; Gestão da assistência de enfermagem; Hipertensão Arterial sistêmica; Diabetes Mellitus; Atenção primária à saúde. 


\begin{abstract}
Objective: To report the experience lived in the planning of educational actions on Hypertension and diabetes carried out with elderly people in primary care, using the conversation wheel strategy. Method: This descriptive study deals with an experience report on the use of the conversation wheel as a strategy for planning health education activity with elderly users, in the context of practical theoretical teaching of nursing management discipline I, in the first half of 2019 in a health unit in the city of Niterói-RJ, linked to the Unified Health System. Results: The conversation wheel is a satisfactory strategy since the target audience living with hypertension and diabetes had the opportunity to expand their perspectives to make better decisions and improve their quality of life resulting from the exchange of knowledge of topics of interest to users. Conclusion: The planning of the activities of the conversation wheel as a strategy to achieve the objectives of promoting the health of hypertensive and diabetic elderly, provided active participation of them, with exchanges of information aimed at the development of the individual's autonomy and the reduction of damages. With regard to students, it brought clarity of their role in the planning and organization of their practice, as well as greater security in their approaches as future professionals responsible for education in user and community health.

Keywords: Health planning; Health education; Nursing care management; Systemic arterial hypertension; Diabetes Mellitus; Primary health care.
\end{abstract}

\title{
Resumen
}

Objetivo: Informar la experiencia vivida en la planificación de acciones educativas sobre Hipertensión y diabetes realizadas con personas mayores en atención primaria, utilizando la estrategia de rueda de conversación. Método: Este estudio descriptivo aborda un informe de experiencia sobre el uso de la rueda de conversación como estrategia para la planificación de la actividad de educación para la salud con usuarios mayores, en el contexto de la enseñanza teórica práctica de la disciplina de gestión de enfermería I, en el primer semestre de 2019 en una unidad de salud en la ciudad de Niterói-RJ, vinculado al Sistema Único de Salud. Resultados: La rueda de conversación es una estrategia satisfactoria ya que el público objetivo que vive con hipertensión y diabetes tuvo la oportunidad de ampliar sus perspectivas para tomar mejores decisiones y mejorar su calidad de vida como resultado del intercambio de conocimientos de temas de interés para los usuarios. Conclusión: La planificación de las actividades de la rueda de conversación como estrategia para alcanzar los objetivos de promoción de la salud de los ancianos hipertensos y diabéticos, proporcionó la participación activa de los mismos, con intercambios de información dirigidos al desarrollo de la autonomía del individuo y la reducción de daños. Con respecto a los estudiantes trajo claridad de su papel en la planificación y organización de su práctica, además de una mayor seguridad en sus planteamientos como futuros profesionales responsables de la educación sanitaria de los usuarios y de la comunidad.

Palabras clave: Planificación de la salud; Educación para la salud; Gestión de la atención de enfermería; Hipertensión arterial sistémica; Diabetes mellitus; Primeros auxilios.

\section{Introdução}

O envelhecimento populacional é uma realidade mundial, e esta revolução demográfica está atrelada a diversos fatores, inclusive os avanços nas tecnologias em saúde. No Brasil, dados do Instituto Brasileiro de Geografia e Estatística (2018), mostra que o número de idosos pode chegar a 25,49\% da população até 2060. Algumas mudanças fisiológicas, fisiopatológicas e sociais que acompanham o processo de envelhecimento, contribuem para o aparecimento de algumas comorbidades como as doenças crônicas, com destaque para a Hipertensão Arterial Sistêmica (HAS) e o Diabetes Mellitus (DM), que constituem os principais fatores de risco para as doenças cardiovasculares, motivo pelo qual representam agravos de saúde pública, destes, cerca de 60 a $80 \%$ dos casos pode ser tratado na rede básica (Brasil, 2001).

Dentro deste contexto a educação em saúde tem papel fundamental na promoção da mesma, na prevenção de doenças e no engajamento da população, possibilitando que a haja maior participação por parte das pessoas em assuntos relacionados à saúde, alcançando assim maior qualidade de vida e redução dos agravos. A educação em saúde é um dos principais dispositivos para viabilizar a promoção da saúde na atenção básica no Brasil. O reconhecimento de que a saúde tem um caráter multidimensional e de que o usuário é um sujeito ativo da educação em busca de autonomia em seu cuidado são condições essenciais à prática neste âmbito da atenção (Vasconcelos et al., 2017).

De acordo com Oliveira (2011), a promoção da saúde consiste nas atividades dirigidas à transformação dos comportamentos dos indivíduos, focando nos seus estilos de vida e promovendo uma modificação na sua maneira de pensar e agir. Para isto, distintas são as estratégias que nos possibilitam uma comunicação efetiva com os diversos públicos que são 
objetos das nossas ações. Tais estratégias são importantes para auxiliar a propagar o conhecimento e unir todas as pessoas que necessitam adquirir as informações necessárias para o seu aprendizado, facilitando a troca entre todos os participantes, como profissionais de saúde e pacientes havendo assim, uma comunicação efetiva onde se estabelece a troca de conhecimentos entre o profissional e usuário de saúde e entre os próprios usuários que compartilham da mesma realidade e do mesmo agravo aumentando assim a contribuição e fomento do conhecimento entre ambas as partes.

Ressalta-se que para obtenção do fortalecimento da autonomia de indivíduos e grupos sociais, não basta apenas informar. Com esse entendimento compreende-se a roda de conversa como um instrumento fundamental e necessário para a construção da interação entre aquele que ensina e aquele que aprende. Enriquecendo juntos com as experiências de vida, gerando mobilização na promoção a saúde (Assis et al (org), 2002, p. 17).

Nesse sentido, o planejamento torna-se extremamente importante para que as diversas atividades possam atingir os objetivos desejados, sendo assim é relevante sua aplicabilidade na roda de conversa, pois é preciso determinar como será feito, considerando os tópicos que serão abordados, a confecção dos materiais educativos e a forma de condução da discussão do assunto abordado. Além disso, é importante ressaltar que o planejamento das ações educativas auxilia o enfermeiro a ter sua atuação mais efetiva e organizada, aumentando assim a qualidade de seu serviço. De acordo com Chavienato (2003), o planejamento figura como a primeira função administrativa, por ser aquela que serve de base para as demais funções, trata-se de um modelo teórico para uma ação futura onde determina os objetivos e detalha os planos necessários para atingi-los de melhor maneira possível.

O gerenciamento e planejamento de atividades compõem o trabalho do enfermeiro nos mais diversos cenários de atuação, com o objetivo de tornar a prática do cuidado mais qualificada e efetiva. No processo de trabalho do enfermeiro observase a articulação entre as esferas assistencial, gerencial, a pesquisa e o ensino. Neste sentido, o planejamento das atividades constitui uma etapa crucial do processo, permitindo preparo e organização de suas ações para uma assistência mais eficaz e assertiva. Para isto, o enfermeiro além de se apropriar das tecnologias duras, como o saber teórico e técnico, também é um profissional caracterizado pelo uso de tecnologias leves, como o acolhimento e cuidado integrado que envolve como principal veículo a comunicação e a escuta ativa, esta última atua como potencializador do seu papel de gerenciador do cuidado.

Diante do exposto o objetivo do presente trabalho é relatar a experiência vivenciada no planejamento das ações educativas sobre Hipertensão e diabetes realizada com idosos na atenção básica.

\section{Metodologia}

Trata-se de um relato de experiência de natureza descritiva, realizado em uma Unidade de Saúde no município de Niterói-RJ vinculada ao Sistema Único de Saúde-SUS, em que são oferecidos diversos serviços, tais como atendimento para gestantes, acompanhamento e tratamento de pessoas com HIV/AIDS e tuberculose, vacinação, sala de curativos, além dos profissionais proporcionarem permanente educação em saúde como parte de suas atividades no dia a dia. A atividades relatada aconteceu no auditório da unidade, com idosos que participavam do grupo de convivência de idosos da instituição.

A presente pesquisa foi realizada no primeiro semestre de 2019, a partir do diálogo e observação da necessidade sobre determinados conhecimentos dos idosos do grupo de convivência, nesse sentido foi imperativo nos organizarmos e nos apropriarmos da temática: hipertensão arterial e diabetes mellitus em idosos. Desta forma, realizou-se o planejamento, a execução e a avaliação da atividade. Para execução da atividade foi utilizada a roda de conversa como forma de construir um espaço de diálogo que nos possibilitou atingir nosso objetivo tais como a expressão e aprendizado em conjunto. Esta ação foi desenvolvida pelos acadêmicos do sexto período da Escola de Enfermagem Aurora de Afonso Costa (EEAAC) da Universidade Federal Fluminense (UFF), como uma das atividades do ensino teórico-prático da disciplina de Gerência em enfermagem I, sob a supervisão docente. 
O estudo seguiu as recomendações da Resolução 510/16 do CONEP. O relato de experiência não apresenta dados de sujeitos participantes, e constitui uma atividade de serviço.

\section{Resultados e Discussão}

A unidade de saúde, local onde foi realizado o ensino teórico prático, possui um grupo de convivência de idosos, composto aproximadamente por 20 idosos, que se reúnem semanalmente, às quartas-feiras, no período da manhã sob a coordenação da assistente social da instituição. Para o desenvolvimento da ação educativa, as acadêmicas dialogaram com a coordenadora do grupo e os usuários idosos sobre o tema que desejariam que fossem abordadas na atividade educativa, desta forma, a temática proposta foi hipertensão arterial sistêmica e diabetes mellitus. A escolha foi de grande relevância, uma vez que indivíduos idosos com hipertensão e diabetes possuem necessidades específicas e limitações, que devem ser gerenciados, para melhora da sua qualidade de vida (Andrade et al., 2012).

A partir da escolha da temática, deu-se início ao planejamento da ação educativa, e discussão das atividades para serem implementadas. Para Lanzoni et al. (2015), a assistência de enfermagem deve ser pautada no planejamento e gerenciamento dos cuidados propostos. A educação em saúde é uma atividade inerente ao profissional de enfermagem, que regulamenta no artigo 11, da lei do exercício profissional, que cabe ao enfermeiro, como integrante da equipe de saúde, realizar educação em saúde visando a melhoria de saúde da população (Brasil, 1986). Então, como parte integrante do processo de cuidado, as atividades educativas devem ser planejadas e organizadas, buscando uma melhor qualidade.

As atividades propostas ao grupo ocorreram em um único encontro, no período de oito as doze horas, utilizando estratégias para estimular a participação ativa dos idosos. A interação dos integrantes do grupo é parte fundamental, e que deve ser valorizada no desenvolvimento de ações, pois permite abordagem de assuntos do seu interesse. Para Mendonça et al. (2013), essa proatividade do público-alvo das intervenções, é o que proporciona uma dinâmica mais produtiva com resultados mais efetivos, com relação a mudança de comportamento e empoderamento em saúde.

O ponto de partida do planejamento foi a escolha da dinâmica de intervenção assim como o objetivo da atividade. Entende-se que a ação educativa deve ter um caráter socioeducativo, ou seja, possibilitar a interação entre os participantes ao mesmo tempo em que proporciona a troca de conhecimentos científico e popular, presente no cotidiano dos idosos (Cabral et al., 2015). Com esse intuito, a roda de conversa foi a dinâmica selecionada, devido a sua fundamentação metodológica, que tem como princípios a horizontalização das relações de poder e ressignificação de saberes de forma dialógica (Sampaio et al, 2014).

A partir da escolha da dinâmica, um segundo momento foi destinado para a seleção dos tópicos mais relevantes para serem abordados com os idosos, durante a ação educativa. A escolha dos tópicos foi baseada nos principais questionamentos percebidos no grupo, e foi limitada pelo tempo que seria disponível para intervenção. Como apoio didático e forma de organizar os tópicos selecionados, as acadêmicas desenvolveram folders que foram disponibilizados para os idosos após a intervenção.

O folder foi planejado e estruturado de forma a incentivar o autocuidado e estimular a percepção de hábitos e comportamentos que os expõem a riscos, assim como mudanças que contribuem para a qualidade de vida dos idosos. As informações inseridas no material foram as seguintes: conceito das comorbidades, como se desenvolvem, principais complicações, tratamentos medicamentoso e não medicamentoso e formas de prevenir as mesmas. É importante salientar que, os conteúdos que constituíram o material foram fundamentados em fontes confiáveis, tais como artigos científicos, manuais disponibilizados pelo Ministério da Saúde e diretrizes da Sociedade Brasileira de Hipertensão Arterial e Diabetes.

O terceiro momento foi destinado para a aferição da pressão arterial e disponibilização do material didático construído. Neste momento, as acadêmicas tiveram a oportunidade de mais uma vez realizar educação em saúde, através do manejo das fragilidades e dificuldades trazidas por cada participante, possibilitando desta forma uma atuação pontual e singular que oferecesse condições para mudanças comportamentais efetivas para garantia do bem-estar individual. Para Borba et al (2020), a 
educação é um dos componentes do tratamento da diabetes mellitus, envolvendo um processo de aprendizagem complexo e que requer adaptação de acordo com as necessidades do indivíduo e de seu contexto de vida. Deste modo no intuito de promover a autonomia e o gerenciamento do DM, dentre as abordagens educativas, destaca-se a metodologia da problematização, orientada pela percepção da realidade, protagonismo e trabalho em grupo.

A atividade educativa foi dividida em três momentos. Na primeira foi realizada uma sessão de alongamento e roda de música, prática que já era estabelecida e realizada em todos os encontros do grupo. Este tipo de atividade estimula a interação entre os participantes, fortalecendo vínculos e socialização entre os idosos. Práticas que consolidam essa interação é importante, uma vez que, o ingresso em grupos de terceira idade, é um marco importante na vida de um idoso, porque substituem momento de solidão, problemático nesta faixa etária, por momentos de construção de amizades e encontros (Andrade et al, 2012).

O segundo momento, foi destinado para a dinâmica da roda de conversa. Esta etapa da atividade teve como fonte norteadora os construtos de Paulo Freire, e foi baseada em uma abordagem participativa e dialógica, visando o empoderamento dos sujeitos envolvidos (Seabra et al, 2019). Este momento foi marcado por grande entrosamento entre os participantes e as acadêmicas, o que proporcionou um importante troca de experiências e saberes.

A partir do diálogo e troca de experiências alguns questionamentos surgiram o que foi aproveitado para elucidar dúvidas e promover saúde, abordando sobre mudanças do estilo de vida, envolvendo hábitos alimentares e atividades físicas, assim como uso adequado das medicações e manejo de possíveis complicações das comorbidades. Para Sousa e Oliveira (2015), a promoção em saúde é essencial em todas as idades, mas para idosos o seu valor é inquestionável. Desta forma, é fundamental criar e incentivar na população idosa a criar hábitos saudáveis, principalmente no que concerne a sua alimentação e exercícios físicos. Para Cotta et al (2009), a educação e a promoção da saúde não se referem à busca de subsídios para que a doença não se estabeleça, uma vez que no portador da diabetes mellitus e hipertensão arterial já se instalou; o enfoque, neste caso, refere-se à importância de que estes indivíduos se conscientizem que é possível levar uma vida saudável e normal, quando se é portador de uma doença crônica. Nesse sentido, a mudança nos hábitos de vida é de fundamental importância, não só para estes usuários da equipe de saúde da família, como também para aqueles que estão ao seu redor, evitando, assim, que indivíduos pré-dispostos ao diabetes e à hipertensão desenvolvam também a doença.

A discussão sobre mudanças do estilo de vida despertou maior interesse do grupo, hábito alimentar saudáveis foi abordado com o intuito de estimular à compreensão dos idosos com relação aos alimentos que eles podem e devem ingerir. Já atividade física foi tratada como elemento complementar indispensável no tratamento não medicamentoso e um importante auxiliador da manutenção da funcionalidade da população idosa. Para Silva et al (2018), a prática de atividade física para os idosos além de diminuir os danos causados pelo sedentarismo, ainda é vista como um método agradável para a conservação da saúde, pois fortalece as relações sociais, traz autoconfiança e favorece a manutenção da autonomia.

O terceiro momento foi destinado para a aferição da pressão arterial e disponibilização do material didático construído. Neste momento, as acadêmicas tiveram a oportunidade de mais uma vez realizar educação em saúde, através do manejo das fragilidades e dificuldades trazidas por cada participante, possibilitando desta forma uma atuação pontual e singular que oferecesse condições para mudanças comportamentais efetivas para garantia do bem-estar individual. Para Borba et al (2020), a educação é um dos componentes do tratamento da diabetes mellitus, envolvendo um processo de aprendizagem complexo e que requer adaptação de acordo com as necessidades do indivíduo e de seu contexto de vida. Deste modo no intuito de promover a autonomia e o gerenciamento do DM, dentre as abordagens educativas, destaca-se a metodologia da problematização, orientada pela percepção da realidade, protagonismo e trabalho em grupo.

Ressalta-se que no decorrer da atividade, observou-se a necessidade de complementar a roda de conversa com a realização da aferição da pressão arterial dos integrantes do grupo, tornando-se um momento de maior integração e interação com cada um dos participantes, o que sem dúvida possibilitou fortalecer o que foi dialogado no coletivo; como por exemplo, 
estimulou-se a prática da aferição como parte do cuidado domiciliar para acompanhamento da comorbidade. Estudos apontam a monitorização residencial da pressão arterial como um método mais apropriado do que a medida de consultório e tão boa quanto à monitorização da pressão arterial, pois promove diagnóstico preciso e melhor acompanhamento da hipertensão ao longo do tratamento (Silva \& Pierin, 2012).

A hipertensão arterial sistêmica (HAS) é uma condição clínica multifatorial caracterizada por níveis elevados e sustentados de pressão arterial, apresenta PA maior ou igual 140 x $90 \mathrm{mmHg}$ (Brasil, 2013). A doença se desenvolve atacando os vasos sanguíneos e evolui para danos cardiovasculares e cerebrovasculares além de outros diversos danos. Dentre os tratamentos medicamentosos temos os anti-hipertensivos que agem controlando a pressão arterial. Nos tratamentos não medicamentosos incluem as estratégias para ensinar o idoso a ter maior qualidade de vida, como por exemplo diminuição de bebidas e uso de cigarros, mudanças nos hábitos alimentares e práticas de exercícios físicos. A prevenção da hipertensão arterial e diabetes melittus é feita principalmente através de bons hábitos alimentares e estilo de vida.

Em relação à hipertensão, o principal agravo relacionado a ela é a crise hipertensiva, porém outras complicações são recorrentes, como problemas cardiovasculares e cerebrovasculares. Desta forma, é importante que seja feito o manejo adequado dessas doenças com práticas de autocuidado para que diminua os riscos de desenvolvimento dessas complicações. O estudo de Santos e Moreira (2012), identificou como fatores de risco o tabagismo, sedentarismo, sobrepeso/obesidade e história familiar de doença cardiovascular, presentes em usuários com hipertensão, diabetes e em pessoas com as duas doenças. Quanto às complicações, encontraram-se coronariopatias, infarto agudo do miocárdio, acidente vascular cerebral e doença renal presentes em usuários com hipertensão, com diabetes e com as duas doenças.

Assim, também é importante atentar ao manejo dessa doença, principalmente em relação à alimentação, é importante que o idoso seja orientado sobre os alimentos e como seus hábitos podem influenciar no agravo da doença e em sua qualidade de vida. Além disso de acordo com Brasil (2013), também são considerados aspectos importantes para o manejo adequado da doença a redução de peso, alimentação saudável, atividade física e moderação no consumo de álcool. Os resultados do estudo de Santos e Moreira et al (2012), expõem a necessidade de que o enfermeiro promova educação em saúde a ser realizada com os usuários, com o fim de amenizar a incidência de complicações.

O manejo dessas doenças se dá principalmente através da educação em saúde, onde o paciente aprende quais são os agravos e como eles podem ser evitados. Assim, é preciso demonstrar através dos encontros como se desenvolvem os agravos das mesmas, tais como a retinopatia que pode causar cegueira permanente, a pele mais sensível que deve sempre ser protegida principalmente nos pés para que não haja lesões, a neuropatia diabética que implica na degeneração dos nervos o que pode ocasionar em perda da sensibilidade e maior probabilidade de desenvolver lesões de difícil cicatrização e provável infecção, a nefropatia diabética que acarreta através de lesões nos rins em seu mal funcionamento, entre outros.

Foi exposto de forma dialógica que a diabetes mellitus é uma síndrome decorrente da falta de insulina e consequentemente a ausência de seus efeitos no corpo causa distúrbios metabólicos como a hiperglicemia. A doença se desenvolve quando o corpo não é mais capaz de controlar os níveis de açúcar no sangue, por conta da diminuição ou ausência da insulina que tem a função de facilitar a entrada de glicose nas células.

O manejo dessas doenças se dá principalmente através da educação em saúde, onde o paciente aprende quais são os agravos e como eles podem ser evitados. Assim, é preciso demonstrar através dos encontros como se desenvolvem os agravos das mesmas, tais como a retinopatia que pode causar cegueira permanente, a pele mais sensível que deve sempre ser protegida principalmente nos pés para que não haja lesões, a neuropatia diabética que implica na degeneração dos nervos o que pode ocasionar em perda da sensibilidade e maior probabilidade de desenvolver lesões de difícil cicatrização e provável infecção, a nefropatia diabética que acarreta através de lesões nos rins em seu mal funcionamento, entre outros.

Desta forma, é importante que seja feito o manejo adequado dessas doenças com práticas de autocuidado para que 
diminua os riscos de desenvolvimento dessas complicações. O estudo de Santos e Moreira (2012), identificou como fatores de risco o tabagismo, sedentarismo, sobrepeso/obesidade e história familiar de doença cardiovascular, presentes em usuários com hipertensão, diabetes e em pessoas com as duas doenças. Quanto às complicações, encontraram-se coronariopatias, infarto agudo do miocárdio, acidente vascular cerebral e doença renal presentes em usuários com hipertensão, com diabetes e com as duas doenças.

No caso da hipertensão também é importante atentar principalmente em relação à alimentação, é importante que o idoso seja orientado sobre os alimentos e como seus hábitos podem influenciar no agravo da doença e em sua qualidade de vida. Além disso de acordo com o Ministério da Saúde (2013), também são considerados aspectos importantes para o manejo adequado da doença a redução de peso, alimentação saudável, atividade física e moderação no consumo de álcool. Os resultados do estudo de Santos e Moreira et al (2012), expõem a necessidade de que o enfermeiro promova educação em saúde a ser realizada com os usuários, com o fim de amenizar a incidência de complicações.

Ao longo das atividades foi possível perceber que os participantes demonstraram bastante interesse nos assuntos abordados, com colocações relacionadas às experiências pessoais no tocante à saúde, possibilitando uma troca que contribuiu significativamente para construção de novos saberes dos idosos, quanto das acadêmicas.

Uma dificuldade encontrada pelas acadêmicas diz respeito à condução do diálogo, devido a pouca experiência no desenvolvimento deste tipo de atividade. De acordo com Seara et al (2019), o planejamento e excussão de ações dialógicas com idosos pode ser desafiador para acadêmicos, isto porque diferente do modelo tradicional, este permite uma abordagem participativa e dinâmica, que requer o desenvolvimento de habilidades para guiar e coordenar as atividades.

A experiência de planejar e ver a execução da roda de conversa com hipertensos e diabéticos foi bastante enriquecedora. A troca de conhecimentos entre pacientes e os estudantes conduziu muito bem as discussões propostas e nos auxiliou a ensinar melhor os pacientes sobre algumas questões. Além disso, seus estilos de vida e suas atitudes em relação às doenças no dia a dia foram considerados importantes e enriquecedoras para a fomentação da conversa e para o ensino de novas práticas e novos conhecimentos.

\section{Considerações Finais}

O presente estudo evidenciou a importância do planejamento de enfermagem para alcançar os objetivos que são propostos, seja na prática do dia a dia dentro das instituições, quanto no processo de ensino-aprendizagem no âmbito acadêmico para os discentes. Participar de ações voltadas para a atenção primária demonstrou o grande papel que o enfermeiro tem frente à sua prática e a importância do gerenciamento do trabalho para o cuidado das pessoas.

O enfermeiro ao realizar prática educativa em uma unidade básica de saúde, precisa ter uma visão ampla, que vai além da abordagem do assunto que será discutido. É preciso considerar a multicausalidade do processo saúde-doença daquelas pessoas, utilizar métodos dialógicos e promover a participação ativa do sujeito a fim de realizar uma troca enriquecedora e consolidar o conhecimento das pessoas assistidas. A educação em saúde é um dos principais dispositivos para viabilizar a promoção da saúde na atenção primária a saúde no Brasil. O reconhecimento de que a saúde tem um caráter multidimensional e de que o usuário é um sujeito da educação em busca de autonomia são condições essenciais à prática nesse âmbito de atenção.

Para atender às perspectivas da prática em educação em saúde foi necessário mobilizar as nossas competências que estamos adquirindo no decorrer de nossa formação, voltando a nossa ótica para a formação de profissionais críticos, reflexivos e criativos, capazes de se comprometer com a construção de uma prática enriquecedora. Para a execução desses quesitos citados foi exigido além do domínio sobre a temática abordada, o conhecimento sobre planejamento, organização, execução e avaliação da atividade educativa. 
O gerenciamento exerce grande importância para a formação, pois além da finalidade em promover a interação e a troca de experiências sobre as questões relacionadas à saúde do idoso, nos possibilitou gerenciar a assistência ao paciente e consequentemente lhe entregar um serviço de qualidade.

\section{Referências}

Andrade, T. P., Mendonça, B. P. C. K., Lima, D. C, Alfenas, I. C, \& Bonolo, P. F. (2012). Projeto conviver: estímulo à convivência entre idosos do Catete, Ouro Preto, MG. Revista Brasileira de Educação Médica, 36(1), 81-85.

Assis, M, Bertholasce, A. C, Menezes, I. S, Pacheco, L. C, Menezes, M. F. G, Cunha, M. C, Rodrigues, R, Guerreiro, T, Silveira, T. M, \& Araújo, T. D.(2002). Promoção da Saúde e Envelhecimento: orientações para o desenvolvimento de ações educativas com idosos.: Crde Unati Uerj.

Borba, A. K O. T, et al (2020). Intervenção educativa problematizadora para promoção de hábitos saudáveis em idosos com diabetes: ensaio clínico randomizado. Revista Brasileira de Enfermagem. 73.

Brasil. Ministério da Saúde. Estratégias para o cuidado da pessoa com a doença crônica: hipertensão arterial sistêmica. Brasília: Ministério da saúde, 2013. (Cadernos de Atenção Básica, n 37).

Brasil. Ministério da Saúde. Estratégias para o cuidado da pessoa com doença crônica: diabetes mellitus. Brasília: Ministério da Saúde, 2013. (Cadernos de Atenção Básica, n 36).

Cabral, J. R, et al (2015). Education workshops in health with elderly: a life quality promotion strategy. Revista Enfermagem Digital Cuidado e Promoção da Saúde, 2(1), 61-65.

Carneiro, A. C. L. L, et al (2012) Educação para a promoção da saúde no contexto da atenção primária. Rev Panam Salud Publica. 31(2):115-120.

Chavienato, I. (2003) Introdução à teoria geral da administração. Elsevier, 2003.

Cotta, R. M. M., et al (2009). Hábitos e práticas alimentares de hipertensos e diabéticos: repensando o cuidado a partir da atenção primária. Revista Nutr. 22 (6). Instituto Brasileiro de Geografia Estatística. (2018). Projeção da população do Brasil e das Unidades da Federação. Brasil

Lazoni, G. M. M., et al (2015).Tornando-se gerente de enfermagem na imbricada e complexa fronteiradas dimensões assistencial e gerencial. Revista Eletrônica de Enfermagem, 17 (2), 322-332.

Lei $\mathrm{n}^{\circ} 7.498$ (1986). Dispõe sobre a regulamentação do exercício da Enfermagem e dá outras providências. Brasília, DF, Brasil. Recuperado de http://www.cofen.gov.br/lei-n-749886-de-25-de-junho-de-1986_4161.html.

Mendonça, E. T, et al (2013). The experience of educational workshops with older people: (re)considering practices in view of Freire's thinkin. Revista de APS, $16(4), 479-484$.

Ministério da Saúde. (2001). Caderno de Atenção Básica Nº 7: Hipertensão arterial sistêmica - HAS e Diabetes mellitus - DM. Brasília: Ministério. Recuperado de https://bvsms.saude.gov.br/bvs/publicacoes/hipertensao_arterial_sistemica_cab7.pdf

Oliveira, WW. (2011) A importância das ações de promoção da saúde realizadas pelo enfermeiro na equipe de saúde da família[monografia].Conselheiro Lafaiete: Universidade Federal de Minas Gerais- UFMG.

Sampaio, J, et al (2014). Limites e potencialidades das rodas de conversa no cuidado em saúde: uma experiência com jovens no sertão pernambucano. InterfaceComunicação, Saúde, Educação,18(2), 1299-1311.

Santos, J. C., \&Moreira, T. M. M. (2012) Fatores de risco e complicações em hipertensos/diabéticos de uma regional sanitária do nordeste brasileiro. Revista da Escola de Enfermagem da USP 46 (5).

Seabra, C. A. M., et al (2019). Health education as a strategy for the promotion of the health of the elderly: an integrative review. Revista Brasileira de Geriatria e Gerontologia, 22(4)

Silva, G. C. A., \& Pierin, A. M. G. (2012) A monitorização residencial da pressão arterial e o controle de um grupo de hipertensos. Revista Escola de Enfermagem USP. 46 (4): 922-928.

Silva, R. S., et al (2018) A importância da atividade física em idosos com diabetes: revisão bibliográfica. Revista diálogos em saúde. 1(2)

Sousa, E. M S., \& Oliveira, M. C. C. (2015). Viver a (e para) aprender: uma intervenção-ação para a promoção do envelhecimento ativo. Revista Brasileira de Geriatria e Gerontologia, 18(2), 405-415.

Vasconcelos, M. I. O., Farias, Q. L. T., Nascimento, F. G., Cavalcante, A. S. P., Mira, Q. L. M., \& Queiroz, M. V. O. (2017) Educação em saúde na atenção básica: uma análise das ações dos hipertensos. Rev. APS. 20(2): 253 - 262.

Westphal, M. F. (2006) Promoção da saúde e prevenção de doenças. Em: Campos GW, org. Tratado de saúde coletiva. São Paulo/Rio de Janeiro: Hucitec/Fiocruz. 